
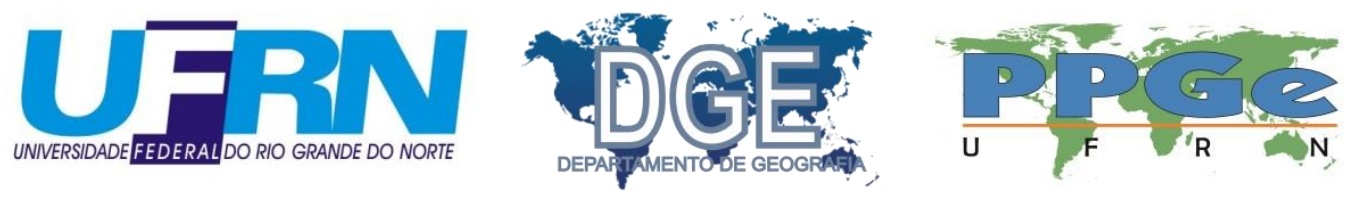

\title{
REVISTA SOCIEDADE E TERRITÓRIO
}

Volume 29 / Número 2 ISSN 2177-8396

\author{
UNIVERSIDADE FEDERAL DO RIO GRANDE DO NORTE \\ CENTRO DE CIÊNCIAS HUMANAS,LETRAS E ARTES \\ DEPARTAMENTO DE GEOGRAFIA \\ PROGRAMA DE PÓS-GRADUAÇÃO E PESQUISA EM GEOGRAFIA
}

\section{EDITOR CHEFE}

Francisco Fransualdo de Azevedo - UFRN - Editor chefe

\section{EDITORES ASSISTENTES}

Igor Rasec Batista de Azevedo - UFRN - Editor Assistente

Joyce Clara Vieira Ferreira - Editor Assistente

Rafael Pereira da Silva - UFRN - Editor Assistente

Regivaldo Sena da Rocha - UFRN - Editor Assistente

\section{CONSELHO EDITORIAL}

Dr. Alexandre Queiroz Pereira - UFC - Brasil

Dr. Antonio Nivaldo Hespanhol - UNESP/PP - Brasil

Dr. Hélsio Amiro Motany de Albuquerque Azevedo - UEM - Moçambique

Dr. Horacio Capel Sáez - UB - Espanha

Dr. José Lacerda Alves Felipe - UFRN - Brasil

Dra. Márcia da Silva - UNICENTRO - Brasil

Dra. Maria Angels Alió - UB - Espanha

Dr. Rodrigo de Freitas Amorim - UFRN - Brasil

Dr. Santiago Linares - UNICEN - Argentina

Dra. Vera Salazar Pêssoa - UFG/Campus Catalão - Brasil

\section{CAPA}

Celso Donizete Locatel - UFRN 
Dr. Ademir Araújo da Costa - UFRN

Dr. Adriano Lima Troleis - UFRN

Dr. Aldo Aloísio Dantas da Silva - UFRN

Dr. Alessandro Dozena - UFRN

Dr. Alexandre Queiroz Pereira - UFC

Dr. Anelino Francisco da Silva - UFRN

Dra. Angela Maria Endlich - UEM

Dr. Antonio Aledo Tur - UA/Espanha

Dr. Antonio Carlos Vitte - UNICAMP

Dr. Antonio Cézar Leal - UNESP/PP

Dr. Antônio José Teixeira Guerra - UFRJ

Dr. Antonio Nivaldo Hespanhol - UNESP/PP

Dra. Carla Silvia Pimentel - UEPG

Dr. Carlos Alberto Feliciano - UNESP/PP

Dr. Celso Donizete Locatel - UFRN

Dr. Cícero Nilton Moreira da Silva - UERN

Dr. Clécio Azevedo da Silva - UFSC

Dr. Clemente Herrero Fabregat - UAM/Espanha

Dra. Diana Lan - UNICEN/Argentina

Dra. Doralice Satyro - UFPB

Dr. Edilson Alves de Carvalho - UFRN

Dr. Edilson Alves Pereira Júnior - UECE

Dra. Edna Maria Furtado - UFRN

Dr. Edson Belo Clemente de Souza - UNIOESTE

Dra. Eliane Tomiasi Paulino - UEM

Dr. Eliseu Savério Sposito - UNESP/PP

Dr. Ermínio Fernandes - UFSCar

Dra. Eugênia Maria Dantas - UFRN

Dra. Eulalia Ribeiro Carbó - UNAM/México

Dr. Evandro César Clemente - UFG

Dr. Everaldo dos Santos Melazzo - UNESP/PP

Dr. Ewerton Vieira Machado - UFSC

Dr. Fernando Moreira da Silva - UFRN

Dr. Francisco Fransualdo de Azevedo - UFRN

Dr. Gleydson Pinheiro Albano - UFRN

Dr. Guillermo Velazquez - UNICEN/Argentina

Dr. Gustavo Buzai - UNLu/ Argentina

Dr. Hélsio Amiro Azevedo - UEM/ Moçambique

Dr. Horacio Capel Sáez - UB/Espanha

Dra. Ideni Terezinha Antonello - UEL

Dra. Ione Rodrigues Diniz Morais - UFRN

Dr. Isonel Sandino Meneguzzo - UEPG

Dra. Jeane Medeiros da Silva - UFRN

Dr. Jeffer Chaparro - UNAL/Colômbia

Dr. João Lima Sant'Anna Neto - UNESP/PP

Dr. João Manoel de Vasconcelos Filho - UFRN

Dr. João Mendes da Rocha Neto - UNB - MI

Dra. Joelma Cristina dos Santos - FACIP/UFU

Dr. José Aldemir Oliveira - UFAM
Dr. José Bueno Conti - USP

Dr. José Omar Moncada Maya - UNAM/México

Dra. Josefa de Lisboa Santos - UFS

Dra. Josefina Di Nucci - UNICEN/Argentina

Dra. Joseli Maria Silva - UEPG

Dr. Jurandyr Luciano Sanches Ross - USP

Dra. Leila Christina Dias - UFSC

Dr. Leonel Brizolla Monastirsky - UEPG

Dra. Lia Osorio Machado - UFRJ

Dr. Luiz Antônio Cestaro - UFRN

Dr. Lutiane Queiroz de Almeida - UFRN

Dr. Magno Vasconcelos P. Junior - UB/Barcelona

Dr. Marcelo dos Santos Chaves - UFRN

Dra. Márcia da Silva - UNICENTRO

Dr. Marco Tulio Mendonça Diniz - UFRN

Dr. Marcos Aurélio Saquet - UNIOESTE

Dra. Margarete Cristiane Trindade Amorim - UNESP/PP

Dra. Maria Angels Alió - UB/Espanha

Dra. Maria Aparecida Pontes da Fonseca - UFRN

Dra. Maria da Encarnação Beltrão Sposito - UNESP/PP

Dra. Maria Francisca de Jesus Lírio Ramalho - UFRN

Dra. Maria Goretti da Costa Tavares - UFPA

Dra. Maria Laura da Silveira - UBA/Argentina

Dra. Maria Mónica Arroyo - USP

Dra. Miriam Hermi Zaar - UB/Portal

Geocrítica/Espanha

Dr. Mozart Fazito Rezende Filho - UNB

Dr. Nildo Aparecido de Melo - IFC

Dra. Núbia Dias dos Santos - UFS

Dr. Orgival Bezerra da Nóbrega Júnior - UFRN

Dra. Patrícia Francisca de Matos - FACIP/UFU

Dr. Paulo César de Araújo - UFRN

Dr. Pere Sunyer Martín - UAM/México

Dra. Rita de Cássia Ariza da Cruz - USP

Dra. Rita de Cássia Conceição Gomes - UFRN

Dra. Rosa Maria Vieira Medeiros - UFRGS

Dra. Rosângela A. Medeiros Hespanhol - UNESP/PP

Dra. Rosemeire Aparecida de Almeida - UFMS

Dra. Rosemeri Melo e Souza - UFS

Dr. Santiago Linares - UNICEN/Argentina

Dr. Sebastião Milton Pinheiro da Silva - UFRN

Dr. Sedeval Nardoque - UFMS

Dra. Silvia Meri Carvalho - UEPG

Dr. Silvio José de Lima Figueiredo - UFPA

Dr. Valdenildo Pedro da Silva - IFRN

Dra. Virginia Cavalcante de Holanda - UVA

Dra. Vera Salazar Pêssoa - UFG

Dr. Wilker de Mendonça Nóbrega - UFRN

Dra. Zuleide Maria Carvalho Lima - UFRN 


\section{Editorial}

1. Pré-textuais. $2-4$

2. Nota editorial 5

\section{Artigos}

1. A disseminação do modo industrial: o exemplo de Caicó, no Rio Grande do Norte Eliseu Saverio Sposito

2. Reestruturação produtiva e seus efeitos sobre as políticas sociais: da universalização à focalização. O caso do estado do Rio Grande do Norte

Rosangela Aparecida de Medeiros Hespanhol.

3. Turismo, mercado imobiliário e conflitos socioespaciais no Nordeste brasileiro Maria Aparecida Pontes da Fonseca e Michael Janoschka.

4. International tourism: modern trends, models and processes

Roberto Paolo Vico

5. A expressão regional das universidades públicas nas cidades médias de Sobral-CE e Parnaiba-PI

Virgínia Célia Cavalcante de Holanda

$.94-112$

6. Aspectos da questão agrária no Brasil

Widson Tainan Ros Martins e Sedeval Nardoque.

7. As perspectivas da economia solidária para o desenvolvimento econômico na $10^{\mathrm{a}}$ região do estado de São Paulo e no Vale do Itajaí/SC

Nildo Aparecido de Melo e Maria Eduarda Correa Boell

8. O cruzeiro galego de Comodoro Rivadavia (Argentina): identidade e territorialização Paulo Roberto Baqueiro BRANDÃO 150-168

9. Um mutirão de vozes, rostos e ações: uma leitura das paisagens da Festa-Romaria de Trindade, Goiás

Eguimar Felício Chaveiro, Ricardo Junior de Assis Fernandes Gonçalves e Helsio Amiro Motany de Albuquerque Azevedo. 169-189 